\title{
Gestão de Pessoas e Inovações Gerenciais: um Estudo Baseado em Cognições de Gesto- res
}

\author{
People Management and Managerial Innovations: a Study Based on Managers' Cogni- \\ tions
}

\author{
Mara Rosalia Ribeiro Silva \\ Universidade Federal do Paraná - UFPR - Brasil \\ mararosalia87@gmail.com \\ ORCID: 0000-0002-9878-694X \\ Ana Paula Moreno Pinho \\ Universidade Federal do Ceará - UFC - Brasil \\ ana.pinho@ufc.br \\ ORCID: 0000-0001-9671-8559
}

Submetido em 02/09/2020; Aprovado em 11/10/2020.

\begin{abstract}
Resumo
Objetivos do estudo: Analisar a relação entre práticas de gestão de pessoas (GP) e implementação de inovações gerenciais a partir das cognições de gestores. Metodologia/abordagem: Mediante abordagem qualitativa, e por meio de entrevistas semiestruturadas, foram ouvidos 16 gestores com auxílio da "técnica das fichas". Posteriormente, foi realizada a análise de conteúdo. Principais resultados: As práticas de GP atuam como facilitadoras das inovações gerenciais implementadas pelos gestores. "Envolvimento de pessoas" e "treinamento, desenvolvimento e educação" foram práticas percebidas como as mais relevantes para a implementação de inovações gerenciais, e "recompensas" foi a menos relevante. Contribuições acadêmicas: Este artigo contribui com o campo da GP estratégica, no sentido de que ao estabelecer um planejamento de longo prazo, alinhado aos objetivos organizacionais, e implementar práticas específicas de gestão, a GP colabora com a geração e implementação de inovações gerenciais. Tais inovações foram colocadas em evidência a fim de esclarecer a concepção dos gestores e obter importantes exemplos de práticas e processos inovadores por eles executados. Destaca-se a contribuição metodológica com o uso da "técnica das fichas" enquanto alternativa para o campo dos estudos organizacionais na compreensão das cognições gerenciais. Contribuições práticas: Este estudo oferece, à GP estratégica, informações para que os gestores invistam em um planejamento estratégico com vistas a priorizar as práticas percebidas como mais relevantes para a implementação de inovações gerenciais, além de incrementar e remodelar aquelas percebidas como menos relevantes.

Palavras-chave: Inovações gerenciais. Práticas de gestão de pessoas. Cognições gerenciais.
\end{abstract}

\section{Abstract}

Study objectives: To analyze the perception of managers about the relationship between people management (PM) practices and the implementation of managerial innovations, from managers' cognitions. Methodology / approach: By qualitative approach, 16 managers were heard through semistructured interviews, using the "cards technique". Content analysis was performed. Main results: PM practices act as facilitators of managerial innovations implemented by managers. "Involvement of people" and "training, development and education" were the practices perceived as the most relevant for the implementation of managerial innovations, and "rewards" was the least relevant. Academic contributions: This article contributes to the field of strategic PM, in the sense that, when PM establishes long-term planning aligned with organizational objectives and implements specific management practices, this collaborates with the generation and implementation of managerial innovations. Managerial innovations were highlighted, seeking to clarify the conception of managers, as well as obtaining the main examples of innovative practices and processes performed by them. The methodological contribution of this article is highlighted with the use of the "cards technique", as an alternative for the field of organizational studies in the understanding of managerial cognitions. Practical contributions: 
This study provides the strategic GP with information so that managers can invest in strategic planning in order to prioritize the practices perceived as most relevant for the implementation of managerial innovations, in addition to increasing and remodeling those perceived as less relevant.

Keywords: Managerial innovations. People management practices. Managerial cognitions.

\section{Introdução}

A abordagem estratégica da Gestão de Pessoas (GP) parte da ideia de que as políticas e práticas de GP estejam alinhadas com as diretrizes estratégicas do próprio negócio (Boxall \& Purcell, 2008; Ulrich, 1998), buscando a construção de uma gestão proativa de pessoas com a finalidade de alcançar vantagens competitivas. No que concerne à inovação, um dos principais desafios atuais da GP estratégica está no estabelecimento de modelos e práticas de gestão em que os indivíduos sejam estimulados a aprimorar conhecimentos, habilidades e atitudes voltados à busca de soluções inovadoras dos problemas organizacionais (Lu, Zhu, \& Bao, 2015). 0 recente interesse em investigar a relação entre práticas de GP e inovação tem estimulado diferentes pesquisas na área (Darwish et al., 2019; Bianchi, Quishida, \& Foroni, 2017; Pradana, Pérez-Luño, \& Fuentes-Blasco, 2020).

Imbricadas nas variadas tipologias de inovação organizacional, as inovações gerenciais (IG) são consideradas fontes vitais de vantagem competitiva (Mol \& Birkinshaw, 2009). As inovações gerenciais estão diretamente ligadas a outros tipos de inovações nas organizações, como em produtos, serviços e processos (Hamel, 2007; Lam, 2006), e representam a criação e o aperfeiçoamento de novas abordagens de execução da atividade gerencial (Damanpour \& Aravind, 2011), além da construção de novos procedimentos e estruturas no sistema administrativo que compõem as organizações (Vaccaro et al., 2012).

A principal relevância das inovações gerenciais para as organizações está em propor e executar alterações no âmbito gerencial, capazes de trazer maior eficiência para práticas e processos gerenciais, além de dar sustentação à gestão organizacional como um todo (Birkinshaw, Hamel, \& Mol, 2008; Mol \& Birkinshaw, 2009). Isso exige dos gestores esforço em constante análise das múltiplas demandas organizacionais, o que resulta na implementação de ações inovadoras, processos gerenciais e arranjos estruturais internos alinhados às perspectivas estratégica e operacional da organização: por um lado, as inovações gerenciais devem estar alinhadas às estratégias organizacionais, com propostas de práticas inovadoras que amparam os objetivos centrais da organização; por outro, as inovações gerenciais devem viabilizar a operacionalização das rotinas que envolvem os negócios da organização (Khosravi, Newton, \& Rezvani, 2019).

Em uma busca bibliográfica acerca das inovações gerenciais no Brasil, verificou-se que esse fenômeno tem sido analisado com ênfase tanto nos processos de implementação de inovações gerenciais em empresas privadas (Bastos et al., 2011; Festa, 2015; Ramos \& Helal, 2010; Schreiber \& Pinheiro, 2016), como na Administração Pública e suas implicações nos melhoramentos dos serviços públicos brasileiros (Deus \& Farias, 2015; Gomes \& Farias, 2016); além da validação de uma escala para medir o grau de importância e de adoção de práticas inovadoras de gestão no contexto das universidades públicas brasileiras (Janissek et al., 2017). Apesar da relevância das inovações gerenciais na dinâmica organizacional, poucos são os estudos empíricos que abordam a influência da GP nessa tipologia de inovação nas organizações, tornando-se pertinente o preenchimento dessa lacuna na literatura (Pinho, Silva, \& Evangelista, 2020).

Assim, diante da pertinência da GP estratégica no campo dos estudos organizacionais e a sua relação com o fenômeno da inovação, delineia-se o objetivo geral desta pesquisa, que é analisar a relação entre práticas de GP e a implementação de inovações gerenciais a partir da cognição de gestores. Para tanto, este estudo foi realizado sob uma abordagem qualitativa, por meio de entrevistas semiestruturadas com gestores de nível estratégico de empresas nacionais de grande porte. As entrevistas foram conduzidas por meio da "técnica das fichas" e os resultados foram analisados pelos parâmetros da análise de conteúdo.

Ressalta-se que, ao adotar a perspectiva cognitivista, buscou-se captar, além da percepção dos gestores, as suas cognições, ou seja, a forma como constroem a realidade em que vivem, dando-lhe significado (Schneider \& Angelmar, 1993). Acredita-se que, por meio do aparato cognitivo utilizado pelo indivíduo, ele seja capaz de promover a sua adaptação ao ambiente organizacional e, assim, agir sobre ele (Fiske \& Taylor, 1991). Tal fato leva à ideia de que os gestores também constroem as suas próprias teorias a respeito do ambiente organizacional e dos trabalhadores. Nesse contexto, o agir 
humano no trabalho é fruto do conhecimento acumulado e das crenças construídas sobre a organização empregadora (Pinho \& Souza, 2019).

Assim, com o estudo das cognições, acredita-se que este artigo contribui com o campo dos estudos organizacionais (e, especificamente, na relação entre GP e a implementação de inovações gerenciais) ao buscar compreender a forma como os gestores percebem a realidade em que vivem, como produzem sentido nessa realidade, como fazem a combinação dos fragmentos do seu conhecimento com os sinais do mundo externo, a fim de produzir soluções aos problemas organizacionais e, ainda, como eles transformam todos os seus processos cognitivos em comportamentos e ações inovadoras (Carvalho, 2004).

Este artigo está dividido nas seguintes seções: após a "introdução", há a "revisão teórica" sobre práticas de gestão de pessoas e inovações gerenciais; em seguida, na seção "método", são explicitadas as informações sobre a natureza da pesquisa e os sujeitos entrevistados, bem como as técnicas e procedimentos de coleta e de análise de dados; posteriormente, na seção "análise de dados", os resultados são descritos e discutidos; e, por fim, a seção "conclusão" trata sobre as principais contribuições, limitações do estudo e sugestões para pesquisas futuras.

\section{Revisão teórica: práticas de gestão de pessoas e inovações gerenciais}

A concepção de GP estratégica tem raízes na visão baseada em recursos (VBR) (Barney, 1991; Penrose, 1959). A proposta central da VBR é que a fonte das vantagens competitivas de uma organização encontra-se primariamente nos recursos e competências por ela desenvolvidos e controlados, mediante análise da forma como são obtidos, combinados, controlados e aplicados os seus recursos. Nesse contexto, as pessoas são consideradas recursos que viabilizam a implementação da estratégia organizacional, sendo consideradas fontes de vantagem competitiva das organizações (Wright \& McMahan, 1992).

O termo "estratégica" no âmbito da GP significa algo crítico para a sobrevivência da organização, ou seja, é um núcleo gestor considerado essencial para o bom desempenho do negócio (Boxall, 2003). Nesse sentido, Legge (2005) ressalta que a aproximação entre a GP e a gestão estratégica organizacional é um processo complexo, que envolve a estruturação de um planejamento conjunto entre gestores de diferentes áreas, a construção de relações de confiança entre eles e o alinhamento na execução de planos de ação traçados. A GP estratégica busca atingir os objetivos estratégicos organizacionais por meio do desenvolvimento de pessoas, incentivando e priorizando ações de aperfeiçoamento do capital intelectual dos trabalhadores, trabalho em equipe e comprometimento dos trabalhadores com a missão, visão e valores da organização (Davenport, Prusak, \& Wilson, 2003).

Para se manterem competitivas, as organizações buscam desenvolver e implementar inovações. A relação entre inovação e GP no ambiente organizacional é latente nos estudos organizacionais (Bianchi, Quishida, \& Foroni, 2017; Darwish et al., 2019; Pinho, Silva, \& Evangelista, 2020; Pradana, PérezLuño, \& Fuentes-Blasco, 2020). Mediante ações específicas da GP, os trabalhadores podem propor melhorias e, também, a criação de novos sistemas de gestão ou projetos de organização, contribuindo para a obtenção de vantagens competitivas da empresa. Dessa forma, faz-se necessário implementar práticas de GP que estimulem os funcionários, facilitem o seu processo de aprendizagem, aperfeiçoem o seu desempenho e orientem as suas decisões para a geração de inovações (Tan \& Nasurdin, 2011).

Dentre as práticas de GP mais relevantes associadas ao contexto brasileiro, validadas na Escala de Políticas e Práticas de Recursos Humanos, destacam-se as seguintes ações: recrutamento e seleção; envolvimento de pessoas; treinamento, desenvolvimento e educação (TD\&E); condições de trabalho; avaliação de desempenho e competências (AD\&C); e recompensas (Demo et al., 2011), como apresentadas no Quadro 1.

Uma das atribuições mais características da GP é o "recrutamento e a seleção" de pessoas, cujo processo tem acompanhado a evolução da própria GP nas organizações, sendo cada vez mais voltado à busca e escolha de pessoas dotadas de múltiplas competências técnicas e comportamentais (Ulrich \& Dulebohn, 2015). Essa tarefa consiste em encontrar pessoas alinhadas com as exigências e os valores da empresa (Coradini \& Murini, 2009) e que possuem competências necessárias para desempenhar atividades pertinentes às funções de certo cargo (Prato Neto et al., 2016). Para garantir que a organização tenha seus resultados otimizados por meio do trabalho dos seus funcionários, é preciso buscar e selecionar pessoas que tenham condições de apresentar boa qualidade no seu desempenho individual (Ekwoaba, Ikeije, \& Ufoma, 2015). Ao contratar as pessoas adequadas ao perfil de vagas em aberto, a 
empresa deve ter como meta economizar custos, como de tempo (evitando retrabalho de procedimentos burocráticos e em treinamentos) e de recursos financeiros (poupando a empresa de possíveis prejuízos relacionados às más decisões tomadas por trabalhadores sem qualificações apropriadas) (Ofori \& Aryeetey, 2011).

Quadro 1 - Práticas de Gestão de Pessoas

\begin{tabular}{|c|l|}
\hline Práticas de GP & \multicolumn{1}{c|}{ Descrição } \\
\hline Recrutamento e seleção & $\begin{array}{l}\text { Candidatar e selecionar pessoas que tenham valores, interesses, expectativas e competências } \\
\text { alinhadas com a organização. }\end{array}$ \\
\hline Envolvimento & $\begin{array}{l}\text { Criar vínculos afetivos com os colaboradores, contribuindo com o seu reconhecimento, relacio- } \\
\text { namento, participação e comunicação. }\end{array}$ \\
\hline TD\&E & $\begin{array}{l}\text { Estimular a contínua aprendizagem, aperfeiçoamento de competências e produção de conhe- } \\
\text { cimentos. }\end{array}$ \\
\hline Condições de trabalho & $\begin{array}{l}\text { Oferecer um ambiente de trabalho que preza pela saúde e segurança dos trabalhadores, além } \\
\text { de acesso à tecnologia. }\end{array}$ \\
\hline AD\&C & $\begin{array}{l}\text { Avaliar o desempenho dos trabalhadores, subsidiando as decisões sobre promoções, planeja- } \\
\text { mento de carreira e desenvolvimento. }\end{array}$ \\
\hline Recompensas & $\begin{array}{l}\text { Recompensar o desempenho e as competências dos colaboradores em termos de remuneração } \\
\text { e incentivos. }\end{array}$ \\
\hline
\end{tabular}

Fonte: Adaptado de Demo et al. (2011).

De acordo com Demo et al. (2011), a prática de GP “envolvimento" está relacionada à criação de ações para desenvolver vínculos afetivos com os trabalhadores, contribuindo com o bem-estar dos indivíduos no sentido de garantir reconhecimento, relacionamento estável, participação nas atividades e fluxo livre de comunicação. Em consonância com essa ideia, Fiuza (2010) explica que o envolvimento remete a um conjunto de práticas de reconhecimento, relacionamento, participação e comunicação executadas pela GP a fim de proporcionar melhor qualidade dos relacionamentos entre a organização e os trabalhadores, bem como auxiliar no processo de integração dos indivíduos da organização para que eles sejam participativos na condução dos negócios.

As práticas de GP relacionadas à TD\&E são essenciais para o aprimoramento profissional dos trabalhadores. Para Rumke et al. (2016), o papel do treinamento é desenvolver competências profissionais imediatas, dando subsídios para a tomada de decisão na sua rotina de trabalho e contribuindo para que ele seja mais inovador e criativo, com uma visão macro dos processos e atividades da organização. 0 papel do desenvolvimento, por sua vez, tem o sentido de preparar o trabalhador para futuros desafios e, para tanto, a GP realiza um planejamento de treinamentos e oportunidades educacionais que visam dar suporte aos trabalhadores.

A prática de AD\&C refere-se à análise do desenvolvimento dos conhecimentos, habilidades e atitudes dos trabalhadores na execução das suas funções nas organizações. Dentre os principais objetivos da avaliação de competências, Kachanakova (2013) destaca que esta prática de GP deve permitir a descoberta e o máximo aproveitamento dos conhecimentos, habilidades e atitudes de cada trabalhador, ou seja, investigar e avaliar como trabalhadores cumprem as suas tarefas e exigências de trabalho e como constroem as suas relações com colegas de trabalho, clientes e outros stakeholders.

Os "sistemas de recompensas" são práticas de GP que representam quaisquer tipos de compensação monetária, não monetária ou psicológica oferecidos pelas organizações aos seus trabalhadores por conta do seu desempenho do trabalho (Bratton \& Gold, 2012). Dutra e Hipólito (2012) explicam que as organizações têm a oportunidade de demonstrar quão valiosos são os seus trabalhadores ao implementar diferentes sistemas de recompensas. No caso de sistemas de recompensas alinhados à implementação de inovações, alguns fatores são relevantes para o sucesso dessa relação, como: apoio dos múltiplos níveis gerenciais; monitoramento próximo e direto da GP e por meio de sistemas de informação; e alinhamento dos outros processos e práticas de GP (como ações de recrutamento de pessoas com perfis inovadores e de treinamentos) (Marx, Soares \& Barros, 2016).

No tocante à prática de GP "condições de trabalho", as questões de segurança no trabalho e de acesso às tecnologias (Demo et al., 2011) são relevantes para garantir a manutenção da qualidade de vida no trabalho (Zanelli, 2010). Horta e Barbosa (2017) salientam que o processo de escolha de novas tecnologias deve estar alinhado às estratégias das organizações, bem como às necessidades do negócio e à possibilidade de investimentos. Segundo os autores, o papel dos gestores na adoção de novas tecnologias e no sucesso da implementação envolve o desenvolvimento de confiança mútua entre gestores e trabalhadores. 
Argumenta-se que, na dinâmica organizacional, o conjunto de práticas de GP dá suporte às inovações organizacionais, gerando, em contrapartida, novas ideias que impulsionam a criação e o aperfeiçoamento de práticas inovadoras de gestão. Inovação é aqui definida como qualquer ideia, prática ou objeto que é percebido como novo para certo contexto organizacional (Rogers, 1995). Dentre as inovações possíveis nas organizações, esta pesquisa pretende focar especificamente nas inovações gerenciais, as quais permitem outros tipos de inovações, como tecnológicas, mas, também, são consideradas relevantes fontes de vantagem competitiva (Mol \& Birkinshaw, 2009).

Não há consenso a respeito do conceito de inovações gerenciais e, portanto, ele ainda se encontra difuso na literatura (Damanpour \& Aravind, 2011; Damanpour, 2014). Para fins deste artigo, todavia, as inovações gerenciais significam a criação e a implementação de novas abordagens para realizar o trabalho da gestão (Damanpour, 2014), que resultam em melhorias na eficiência operacional e desempenho organizacional (Lin \& Su, 2014). Em suma, as inovações gerenciais ocorrem no âmbito organizacional e possuem ênfase em três principais perspectivas (Lopes, 2017).

Quanto à primeira perspectiva, salienta-se que as práticas gerenciais compõem o conjunto de decisões tomadas pelas organizações com o fim de conduzir todas as pessoas e departamentos ao alcance de seus objetivos organizacionais estratégicos, sendo que cada setor possui suas práticas segundo a natureza de suas atividades (Cunha, Palma \& Santos, 2008). No caso de práticas gerenciais inovadoras na gestão, elas devem significar o rompimento radical ou incremental dos princípios, processos e ações gerenciais que caracterizam o modelo de gestão tradicional de certo contexto organizacional (Birkinshaw, Hamel, \& Mol, 2008; Hamel, 2007).

A segunda perspectiva está relacionada às inovações gerenciais que possuem foco nos processos gerenciais. Segundo Teece, Pisano e Shuen (1997), os processos gerenciais significam a maneira como as empresas realizam as atividades organizacionais, as quais são relacionadas às rotinas, assim como aos padrões de trabalho e de aprendizagem. Considera-se que novas técnicas de gestão devem ser implementadas (Volberda, Van Den Bosch, \& Heij, 2013; Birkinshaw, Hamel, \& Mol, 2008), assim como novos fluxos de atividade em planejamento estratégico, comunicação interna e gestão do conhecimento (Hamel, 2007), novos procedimentos e, consequentemente, novas rotinas operacionais (Armbruster et al., 2008).

No que tange à terceira perspectiva - inovações gerenciais focadas nas estruturas organizacionais - a ideia está de acordo com a perspectiva de Lam (2006), para quem existem características estruturais que permitem e facilitam novas ideias, resultando em inovações nos produtos e processos do negócio. Essas inovações, quando propostas, trazem alterações e provocam melhorias na distribuição de responsabilidades, em accountability (ou responsabilização), linhas de comando, fluxos de comunicação, número de níveis hierárquicos e divisão de funções (Armbruster et al., 2008). Cruz, Frezatti e Bido (2015) defendem a existência de um sistema de estruturas formais - com controles e indicadores de inovação - que podem gerar o amadurecimento das atividades de inovação.

As práticas de GP no contexto estratégico estão relacionadas às inovações gerenciais. 0 intuito desta pesquisa, portanto, é desvelar a forma como se dá essa relação de acordo com o que pensam os gestores, que são as pessoas que vivenciam inovações nas rotinas da dinâmica organizacional. 0 método escolhido para investigar as nuances dessa relação consta a seguir.

\section{Método}

Esta pesquisa adotou a abordagem qualitativa (Denzin \& Lincoln, 2006). 0 caminho metodológico trilhado para articular o objetivo desta pesquisa está baseado na ideia de que os próprios agentes das inovações (os gestores) podem lançar pistas sobre a forma como estruturam as suas cognições para compreender o fenômeno estudado. Salienta-se que trabalhar com base nas cognições dos gestores significa captar as representações das informações por eles processadas sobre a temática em análise (Schneider \& Angelmar, 1993).

Quanto aos sujeitos da pesquisa, foram entrevistados 16 gestores. A escolha dos sujeitos foi realizada por meio da técnica de "Bola de Neve", segundo três critérios: que eles tivessem experiência em cargos de gestão em nível estratégico; que fossem provenientes de empresas consideradas de grande porte do Estado do Ceará, Brasil - ou seja, empresas que empregam 100 ou mais funcionários no setor de comércio e serviços, e 500 ou mais funcionários no setor industrial (SEBRAE, 2013); e que essas empresas tivessem um departamento de GP estruturado. Por meio desses critérios - e no intuito de buscar uma ampla visão sobre as cognições dos gestores acerca do fenômeno investigado - conside- 
rou-se pertinente entrevistar gestores com diferentes especializações e experiências em variados ramos de atividades. Dentre os 16 gestores entrevistados, seis são do departamento de GP e 10 são de diferentes áreas organizacionais. 0 perfil dos sujeitos da pesquisa está descrito no Quadro 2.

Quadro 2 - Perfil dos sujeitos da pesquisa

\begin{tabular}{|c|c|c|}
\hline $\begin{array}{c}\text { Gestor } \\
\text { entrevistado }\end{array}$ & Cargo & Ramo de atividade \\
\hline E1 & Coordenador pedagógico & Instituição de ensino superior \\
\hline E2 & Gerente administrativo & Transportadora de pessoas e cargas \\
\hline E3 & Editor executivo & Comunicação \\
\hline E4 & Diretor administrativo & Escritório contábil \\
\hline E5 & Gerente de operações & Indústria de alimentos \\
\hline E6 & Engenheiro de controle de qualidade & Indústria de eletrodomésticos \\
\hline E7 & Analista socioambiental & Shopping de varejo \\
\hline E8 & Gerente de vendas & Indústria de alimentos \\
\hline E9 & Coordenador de Departamento Pessoal & Instituição de ensino fundamental e médio \\
\hline E10 & Editor executivo & Empresa de comunicação \\
\hline E11 & Gestor de GP & Varejo da construção civil \\
\hline E12 & Gestor de GP & Transportadora de pessoas e cargas \\
\hline E13 & Gestor de GP & Construtora e imobiliária \\
\hline E14 & Gestor de GP & Instituição de ensino fundamental e médio \\
\hline E15 & Gestor de GP & Serviço hospitalar \\
\hline E16 & Gestor de GP & Indústria de calçados \\
\hline
\end{tabular}

Fonte: Elaborado pelas autoras (2020).

Para a coleta dos dados foram realizadas entrevistas semiestruturadas. Foi solicitada aos participantes a permissão de gravação das entrevistas concedidas para transcrição literal e posterior análise. A duração do tempo total de gravação foi de aproximadamente 609 minutos. 0 roteiro de entrevistas foi dividido em três etapas, sendo a primeira realizada de forma a coletar dados pessoais e profissionais dos participantes.

A segunda etapa teve o intuito de analisar, na ótica dos gestores, a forma como as práticas de GP possibilitam a implementação de inovações gerenciais. Foi utilizado o procedimento metodológico introduzido por Pinho (2009), intitulado "técnica das fichas". Optou-se pela utilização dessa técnica por julgá-la adequada para acessar as cognições dos entrevistados. Acredita-se que quando o pesquisador apresenta as fichas - e solicita que elas sejam comentadas e, posteriormente, ordenadas - o entrevistado é levado a refletir e organizar os seus pensamentos a respeito do conteúdo posto em cada ficha, sendo capaz de compartilhar a forma como significa as suas vivências pessoais e profissionais sobre os conteúdos específicos das fichas (Pinho, 2009). Assim, a "técnica das fichas" baseia-se no livre discurso do entrevistado, que é direcionado a se manifestar de acordo com as palavras-chave presentes nas fichas que, por conseguinte, levam o pesquisador a acessar as cognições dos entrevistados (Pinho, 2009). Assim, foram elaboradas seis fichas contendo palavras que representam ações de GP: recrutamento e seleção; envolvimento; TD\&E; AD\&C; sistemas de recompensas; condições de trabalho (segurança no trabalho e acesso à tecnologia). À medida que as fichas foram apresentadas, questionouse a forma como os conteúdos daquelas fichas estariam relacionados às inovações do gestor.

Na terceira etapa, que teve o objetivo de identificar as práticas de GP mais relevantes para a implementação de inovações gerenciais, solicitou-se aos gestores que colocassem as fichas em ordem de importância - da maior para a menor - levando em conta a que mais possibilita as suas inovações gerenciais. Em seguida, os gestores foram encorajados a justificar aquela ordenação.

As informações coletadas nas entrevistas foram analisadas por meio da análise de conteúdo (Bardin, 2011), dividida em três fases. Na primeira fase - a pré-análise - foi realizada a sistematização das ideias iniciais mediante a transcrição dos áudios e, em seguida, foram repetidas as leituras das transcrições das entrevistas. Nesse momento, percebeu-se que havia a clara divisão dos gestores em dois grupos, os quais foram nomeados "gestores de equipes" e "gestores de pessoas". Essa separação ocorreu em função do seu posicionamento na organização, revelando perspectivas distintas sobre o fenômeno estudado.

Na segunda fase de análise, a exploração do material incorreu na codificação, classificação e categorização dos conteúdos. Foram definidas três dimensões de análise com base nas principais temáticas que orientaram a pesquisa: "papel da GP nas organizações"; "significados de inovação gerencial"; e 
"práticas de GP e implementação de inovações gerenciais". Em seguida, foram determinadas 12 "unidades de contexto", distribuídas de acordo com os conteúdos das dimensões de análise e extraídas dos próprios conteúdos evocados nas transcrições das entrevistas. A unidade de contexto "práticas de GP e implementação de inovações gerenciais" foi subdividida em 14 "unidades de registro", também categorizadas a partir dos conteúdos evocados nas entrevistas. 0 Quadro 3 apresenta a síntese da categorização da segunda etapa da análise de conteúdo.

Quadro 3 - Codificação, classificação e categorização dos conteúdos

\begin{tabular}{|c|c|c|}
\hline Dimensões de análise & Unidades de contexto & Unidades de registro \\
\hline \multirow{2}{*}{ Papel da GP nas organizações } & $\begin{array}{c}\text { Suporte ao planejamento estratégico da } \\
\text { organização }\end{array}$ & 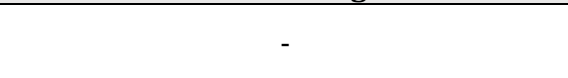 \\
\hline & $\begin{array}{c}\text { Gestores organizacionais são gestores de } \\
\text { pessoas }\end{array}$ & - \\
\hline \multirow{4}{*}{$\begin{array}{l}\text { Significados de inovação } \\
\text { gerencial }\end{array}$} & Nova abordagem para práticas e processos & - \\
\hline & Melhoria contínua de processos & - \\
\hline & Ações que agregam valor à gestão & - \\
\hline & Criatividade da gestão & - \\
\hline \multirow{14}{*}{$\begin{array}{l}\text { Práticas de GP e implementação } \\
\text { de inovações gerenciais }\end{array}$} & \multirow[t]{2}{*}{ Recrutamento e seleção } & $\begin{array}{l}\text { Valores pessoais semelhantes aos valores } \\
\text { da empresa }\end{array}$ \\
\hline & & Seleção por competências \\
\hline & \multirow{2}{*}{ Envolvimento de pessoas } & Intercâmbio de conhecimentos \\
\hline & & Insights para a solução de problemas \\
\hline & \multirow{2}{*}{ Treinamento, desenvolvimento e educação } & Compartilhamento de ideias \\
\hline & & Interação com novos modelos de gestão \\
\hline & \multirow[b]{2}{*}{ Avaliação de desempenho e competências } & Oportunidade de avaliação da carreira \\
\hline & & $\begin{array}{l}\text { Identificação de melhorias no seu } \\
\text { próprio desempenho }\end{array}$ \\
\hline & \multirow{3}{*}{ Sistema de recompensas } & Reconhecimento simbólico \\
\hline & & $\begin{array}{l}\text { Dificuldade de recompensar de forma } \\
\text { justa }\end{array}$ \\
\hline & & $\begin{array}{l}\text { Alinhamento de recompensas com } \\
\text { diferentes ações }\end{array}$ \\
\hline & \multirow{3}{*}{ Condições de trabalho } & Incorporação de novas tecnologias \\
\hline & & Promoção de agilidade nos processos \\
\hline & & Acesso à informação de qualidade \\
\hline
\end{tabular}

Fonte: Elaborado pelas autoras (2020).

Na terceira fase da análise houve tratamento, inferência e interpretação das informações, concretizando-se uma análise crítica e reflexiva do material coletado. A análise das entrevistas e a discussão dos resultados são apresentadas nas seções a seguir.

\section{Análise dos resultados}

A análise da percepção dos gestores acerca da relação entre práticas de GP e a implementação de inovações gerenciais partiu, inicialmente, da compreensão dos gestores entrevistados acerca do significado da GP nas organizações, além do entendimento dos participantes acerca das inovações gerenciais. Por último, foi analisada a percepção dos gestores sobre as práticas de GP e a forma como elas estão relacionadas às próprias inovações no exercício da função gerencial.

\subsection{Cognições acerca do significado da GP e das inovações gerenciais}

A princípio, ao refletir sobre o papel da GP nas organizações, os gestores de equipes e de pessoas destacaram que a dinâmica significa a existência de um departamento que dá suporte ao planejamento estratégico da organização, promovendo-a a um patamar de tomadas de decisões essenciais que definem os rumos que a empresa deseja seguir e o seu posicionamento no mercado. 0 gestor E13 relatou: "[...] a GP tem um papel fundamental em perceber as pessoas e seus potenciais, e como utilizar essas pessoas da melhor forma possível, agregar, e fazer com que elas realmente gerem um retorno 'pra' empresa na forma de trabalho delas".

Ao questionar se os gestores de equipes também se consideram gestores de pessoas, a resposta foi unânime e positiva. Os entrevistados defenderam que eles mesmos executam algumas das atividades tipicamente atribuídas à GP, como seleção de pessoas, motivação da equipe, atividades de integra- 
ção do seu grupo de trabalho, aplicação de treinamentos e gerenciamento de conflitos. Por conta disso, na compreensão dos gestores de equipes, a função de gestor de pessoas está implícita na sua função de gestor de equipes.

Em seguida, ao serem interrogados sobre o que vem à mente ao falar sobre "inovações gerenciais", quatro gestores de equipes evocaram que as inovações se referem a uma nova abordagem sobre práticas e processos na sua gestão, as quais já são executadas na dinâmica da empresa. Sobre isso, o gestor E2 explanou: "para mim, às vezes, não é você trazer algo novo, mas uma compreensão mais esclarecedora, muitas vezes até algo que você já sabia, e aí você faz algo que era velho virar novo".

Para o gestor de equipes E7, essa nova maneira de conduzir a gestão deve ter foco na melhoria contínua dos processos. Já o gestor de equipes E6 destacou que as inovações gerenciais devem agregar valor à gestão, isto é, as mudanças promovidas pelas inovações dos gestores devem trazer melhorias substanciais às operações e às pessoas envolvidas na gestão.

Quanto aos gestores de pessoas, três deles identificaram que inovações gerenciais se referem à criatividade na gestão, ou seja, o gestor ter um perfil criativo e a iniciativa de implementar suas ideias inovadoras. Nesse sentido, mencionou o participante E14: "Para mim inovação gerencial é isso, é mudança, é criatividade, é você buscar fazer o seu trabalho de uma forma criativa e que atenda o resultado". Em contrapartida, na visão de outros dois gestores de pessoas, inovações gerenciais representam a possibilidade de implementação de novas ideias na gestão, ou seja, o gestor deve criar um ambiente aberto para que ele e seu grupo de trabalho possam sugerir ideias e, caso seja viável, permitir que algumas dessas ideias sejam implementadas.

\subsection{Cognições acerca das práticas de GP e a implementação de inovações gerenciais}

A partir da concepção inicial acerca da GP e de inovações gerenciais, os gestores foram levados a refletir sobre a relação entre as práticas de GP e as suas implicações nas próprias inovações enquanto gestores. Sobre esse assunto, torna-se pertinente destacar que, sob a perspectiva dos gestores entrevistados, as práticas de GP devem estar alinhadas ao planejamento estratégico da organização e, por conseguinte, as inovações geradas por eles e por influência das ações diretas da GP resultam na colaboração de todos para o alcance dos objetivos organizacionais.

Os participantes foram unânimes em perceber as seis práticas de GP investigadas neste estudo como facilitadoras das suas próprias práticas gerenciais inovadoras, mas que outros fatores certamente colaboram com a implementação dessas inovações, como a capacidade inovadora da empresa, a disponibilidade e parceria das equipes de trabalho nos processos de inovação e possíveis fatores externos capazes de catalisar os processos de implementação de inovações - como o surgimento de novas tecnologias e de demandas específicas do mercado.

Ao longo do discurso dos gestores, foi possível identificar e pontuar as principais inovações gerenciais por eles implementadas como resultantes das práticas de GP. Especialmente para os gestores de pessoas, a prática de GP "recrutamento e seleção" os tem encorajado a investir em inovações nos modelos e técnicas de seleção de pessoas, com vistas a contratar trabalhadores com experiência em inovações e que demonstrem atitude e comportamento inovador para assumirem os cargos de gestão.

Além disso, os gestores destacaram que as práticas de GP "envolvimento de pessoas", "TD\&E", "AD\&C" e "sistema de recompensas" permitiram a criação e implementação de relevantes inovações no exercício da sua gestão. Uma delas foi a organização e a aproximação das redes de relacionamentos dos gestores, tanto das redes internas - entre outros gestores e as suas próprias equipes de trabalho, por meio da realização de atividades lúdicas e interativas entre os trabalhadores (passeios para cinema, parques) e ações de reconhecimento simbólico que premiaram as lideranças e/ou suas equipes pelos resultados das inovações (vouchers de viagem e de restaurantes, crédito para a participação de curso) -, como das redes externas à organização - com gestores do mesmo ramo de atividade, mas de outras empresas (benchmarking, busca de novas parcerias, eventos corporativos de integração e desenvolvimento de pessoas, palestras com especialistas).

As práticas de GP "envolvimento de pessoas" e "condições de trabalho", por sua vez, incentivaram os gestores a inovar no aperfeiçoamento da gestão participativa entre as suas equipes, priorizando a descentralização das decisões, a sistemática de reuniões com brainstorms, a gestão da diversidade e composição de equipes multifuncionais (compostas por profissionais com diferentes níveis de experiência, idade, escolaridade), além da redistribuição e divisão de tarefas. Ademais, por meio da implementação da prática de GP "condições de trabalho", os gestores refletiram sobre a inovação de melho- 
rias na administração do tempo com vistas à otimização do trabalho das equipes, especialmente por meio da automatização de instrumentos de planejamento e controle de metas e resultados.

\subsubsection{Recrutamento e seleção}

Segundo o discurso dos gestores entrevistados, a prática de GP "recrutamento e seleção" pode atuar como facilitadora de inovações por parte dos gestores na medida em que identifica trabalhadores que tenham valores pessoais semelhantes aos da empresa e que, consequentemente, possuem melhores condições de acompanhar as demandas de inovação da organização. Nessa perspectiva, o gestor E8 relatou: "Eu acredito que esta etapa tem condições de dar este resultado de inovação, porque você vai recrutar as pessoas que são as melhores, que têm os melhores perfis, e que automaticamente são mais inovadoras".

Segundo o entrevistado E5, a realização de uma seleção por competências facilita a entrada de novos gestores com potencial criativo que estimulem a criatividade na gestão, fomentando inovações gerenciais nas organizações.

\subsubsection{Envolvimento de pessoas}

A prática de GP "envolvimento de pessoas", por sua vez, está relacionada às inovações gerenciais, principalmente pela ideia de intercâmbio de conhecimentos. 0 gestor E10 enfatizou que a troca de conhecimentos entre outros gestores pode catalisar as inovações promovidas pela gestão: "Eu acredito que quanto mais gente está junto, quanto mais pessoas pensam sobre determinados processos, contribuem e refletem sobre isso, mais possibilidades têm de haver inovação". Para o gestor de pessoas E14, quando as pessoas são expostas a trocas de conhecimentos, são proporcionados momentos em que os gestores podem ter insights para a solução de problemas - seja do seu departamento ou de outros núcleos na empresa.

No que concerne à relevância das práticas de GP na implementação de inovações gerenciais momento posterior em que os gestores foram convidados a ordenar as práticas de GP, levando em conta quais delas seriam mais relevantes para a implementação das suas inovações gerenciais, gestores de equipes apontaram três vezes a prática "envolvimento de pessoas" em 1ํ lugar. A prática também apareceu sete vezes dentre as quatro primeiras posições. A justificativa dos gestores é que o fator humano impulsiona inovações, e que as pessoas devem estar envolvidas no trabalho e nos objetivos da sua equipe - e também nos objetivos organizacionais - para que as suas ações resultem em inovações.

\subsubsection{Treinamento, desenvolvimento e educação}

De acordo com os gestores entrevistados, a prática "TD\&E” está associada às inovações gerenciais no sentido de permitir o compartilhamento de ideias, bem como entrar em contato com novos modelos de gestão, trazendo adaptações e inovações incrementais ao seu contexto organizacional. $\mathrm{Na}$ opinião de três gestores de equipes, o investimento em ações de TD\&E proporciona a troca de conhecimentos com outros colegas de trabalho ou com os facilitadores do curso, constituindo-se em fontes de ideias e de aprendizado. Nesse contexto, destaca-se a fala do gestor E14:

Eu acredito que este [treinamento, desenvolvimento e educação] talvez seja o tripé essencial para a questão da inovação [...]. O que eu tenho visto é que os processos inovadores são inclusive insights de processos de treinamentos ou de qualificação profissional, educação, conhecimento. Quanto mais capacitado e educado você é, mais capacidade de inovar.

Não por acaso, ao ordenar as práticas de GP na última fase das entrevistas, os gestores de equipes demonstraram a importância da prática “TD\&E” na execução das suas inovações gerenciais, posicionando-a duas vezes em $2^{\underline{0}}$ lugar e cinco vezes entre a $1^{\text {a }}$ e $4^{\text {a }}$ colocação. A relevância dessa prática de GP é que as ações de treinamento e desenvolvimento de pessoas auxiliam no processo de inovações gerenciais, principalmente porque a empresa estimula o trabalhador a focar o seu olhar no ambiente interno (tornando-o mais preparado para sugerir novas ideias à solução de problemas organizacionais) e, ao mesmo tempo, a olhar o ambiente externo (buscando novas ideias em diferentes modelos de gestão aplicados em outros contextos).

\subsubsection{Avaliação de desempenho e competências}

No que tange à prática $\mathrm{AD} \& \mathrm{C}$, os entrevistados relataram que avaliações periódicas podem ser facilitadoras de suas inovações no âmbito gerencial, pois representam oportunidades relevantes de 
avaliação da sua carreira. Isto é, os gestores são capazes de fazer uma análise do status da sua própria carreira e, a partir desse diagnóstico, buscar caminhos para melhorar a sua performance profissional. 0 gestor E5 expressou o seguinte ponto de vista:

o gestor precisa se enxergar, saber o status quo dele para saber para aonde ir. Se você é um gestor e nunca recebeu um feedback, nunca teve uma avaliação de desempenho, não tem como você criar, não tem como você ter a liberdade de se reinventar ou criar alguma coisa, se você nem se conhece.

Segundo o gestor E15, por mais que o feedback seja negativo, é papel da gestão fazê-lo para proporcionar ao gestor a possibilidade de se desenvolver profissionalmente: "Quando você clarifica aquilo que ele precisa melhorar no ponto de vista da empresa a gente está dando a oportunidade de ele querer desenvolver".

\subsubsection{Sistema de recompensas}

A prática de GP "sistema de recompensas", de acordo com os entrevistados, pode promover inovações gerenciais ao trazer reconhecimento simbólico como recompensa pelo serviço prestado pelos trabalhadores. Os gestores de pessoas E13 e E15 concordam que as recompensas financeiras não estão necessariamente ligadas à inovação, mas, sim, às recompensas simbólicas, que são utilizadas pela GP como forma de gratificação e valorização pelo esforço do gestor. Dois gestores de equipes reportaram a dificuldade de premiar de forma justa a implementação de um sistema de recompensas que vise interferir no comportamento inovador de gestores. 0 gestor E3 mencionou que o problema pode estar ligado à dificuldade que a GP tem de mensurar inovações produzidas pelos gestores, assim como não existe métrica para avaliar a influência da ação do gestor naquela inovação.

Não coincidentemente, na fase final das entrevistas, ao posicionarem as seis práticas de GP, os gestores de equipes e de pessoas atribuíram à prática "sistema de recompensas" os últimos lugares. Para os gestores de equipes, as recompensas têm o seu valor no sentido de promover um reconhecimento da performance inovadora do gestor, mas que a efetividade da prática "sistema de recompensas" não se apresenta como essencial à implementação de inovações gerenciais. Sobre essa prática, 0 gestor E7 comentou:

O sistema de recompensa eu acredito que seja menos importante no processo de inovação, porque eu acho que você não tem que fazer porque vai receber um presente, mas porque é fundamental no seu trabalho.

Por sua vez, os gestores de pessoas complementaram que as diversas premiações, por si só não são determinantes aos processos inovadores na gestão, embora sejam valorizados, de forma simbólica, os reconhecimentos dos gestores e suas equipes com performances inovadoras.

\subsubsection{Condições de trabalho}

A última prática de GP analisada foi "condições de trabalho", percebida como alinhada às inovações gerenciais no sentido de que a incorporação e o aprendizado no uso de novas tecnologias estão associados ao fomento de suas inovações gerenciais. Na visão do gestor E10, a GP tem papel essencial no acompanhamento dos grupos de trabalho quanto à absorção e domínio de novas tecnologias - como sistemas e softwares - para que haja o nivelamento dos níveis de conhecimento sobre a tecnologia. 0 gestor E5 salientou que a promoção de agilidade nos processos gerenciais está ligada à implementação de inovações gerenciais, salientando que com mais automatização o gestor fica com tempo livre para realizar outras atividades relacionadas à sua gestão, focando mais em ações estratégias e menos em atividades burocráticas. A seguir, segue a discussão e a síntese dos resultados sobre o fenômeno ora analisado.

\subsection{Discussão e síntese dos resultados}

A partir da análise das cognições dos gestores entrevistados foi possível compreender a relação estabelecida entre as práticas de GP e a implementação de suas inovações gerenciais. A investigação sobre as cognições gerenciais é uma maneira de compreender a realidade à volta do gestor, descrita por meio das suas estruturas cognitivas (Schneider \& Angelmar, 1993). Além disso, analisar a forma como o gestor constrói as suas representações - ou estruturas cognitivas - pode auxiliar na materialização do fluxo dos processos organizacionais, contribuindo para a identificação de fatores que incidem nas inovações gerenciais, bem como no conhecimento dos gargalos do sistema que impedem o fluxo inovador (Schneider \& Angelmar, 1993). Assim, ao conhecer os códigos que formam a construção do 
pensamento que orientam as ações dos gestores, torna-se viável o entendimento do agir organizacional em sua complexidade, o que pode gerar subsídios ao planejamento de políticas e práticas da GP estratégica.

A partir do discurso dos gestores entrevistados, infere-se que a relação entre práticas de GP e a implementação de inovações gerenciais perpassa três níveis de análise na dinâmica organizacional: macro (organizacional), meso (entre as lideranças e suas equipes) e micro (individual). Primeiramente, essa relação ocorre no nível macro, no sentido de que a GP, sob uma abordagem estratégica, tem a função de estruturar, planejar e acompanhar os objetivos de longo prazo concernentes ao trabalho e ao posicionamento das pessoas na organização. Sendo assim, as ações inovadoras promovidas pelos gestores devem estar alinhadas à própria estratégia organizacional. Para tanto, a GP estratégica investe, principalmente, no desenvolvimento dos líderes (Davenport, Prusak \& Wilson, 2003) e na aproximação dos gestores de diferentes áreas com vistas à construção de um plano de ação inovador em comum (Legge, 2005).

Em segundo lugar, a relação entre as práticas de GP e a implementação de inovações gerenciais acontece no nível meso, na medida em que o conjunto de práticas de GP cria um ambiente organizacional propício à criação e à implementação de novas práticas e processos inovadores (Damanpour, 2014), os quais se manifestam na figura das lideranças em constante atividade com suas equipes de trabalho.

Em terceiro lugar, a relação entre as práticas de GP e a implementação de inovações gerenciais intercorre no nível micro, pois é necessário que os gestores individualmente apresentem atitudes e comportamentos orientados às inovações em sua atividade gerencial. Ou seja, a partir das condições organizacionais e das práticas implementadas pela GP estratégica, os gestores colocam em prática os seus conhecimentos, habilidades e atitudes voltados à busca de soluções inovadoras para problemas organizacionais (Lu, Zhu, \& Bao, 2015).

Nesse último nível, referente ao âmbito individual, os gestores particularmente puderam expressar as inovações mais relevantes implementadas em sua própria gestão, que foram, principalmente, ações ligadas à administração do tempo - com vistas à otimização do trabalho das equipes, organização e aproximação das redes de relacionamentos interna e externa à organização e aperfeiçoamento da gestão participativa -, priorizando a gestão da diversidade e a composição de equipes multifuncionais, redistribuição e divisão de tarefas. Percebe-se, inicialmente, que a visão dos gestores acerca das inovações gerenciais coaduna com o que definiu Rogers (1995) sobre inovação, que é definida como qualquer ideia, prática ou objeto percebido como novo em certo contexto organizacional, inferindo que as inovações citadas pelos gestores não representam necessariamente ideias disruptivas para todo o contexto organizacional, mas para a sua conjuntura situacional.

A noção de "criatividade" e de "novas ideias" está no cerne da definição das inovações gerenciais defendida por Damanpour (2014), para quem a gestão deve buscar promover novas abordagens, estratégias e estruturas organizacionais inovadoras. Nesse sentido, a execução de práticas inovadoras na gestão exige um esforço de criatividade e um fluxo de novas ideias por parte dos gestores. A compreensão de que as inovações gerenciais devem ter resultado na agregação de valor e na performance da organização corroboram com o que defendem Birkinshaw, Hamel e Mol (2008), de que as inovações gerenciais devem ter novas abordagens capazes de aprimorar a performance da firma.

Os conteúdos evocados pelos gestores reforçam a ideia de que as ações específicas da GP estratégica colaboram para a geração de inovações. Nesse contexto, os gestores entrevistados perceberam as seis práticas de GP investigadas neste estudo como facilitadoras das suas inovações gerenciais, pois: identificam pessoas que tenham valores voltados à inovação e às competências criativas (Ekwoaba, Ikeije, \& Ufoma, 2015); motivam trabalhadores e promovem intercâmbio de conhecimentos, produzindo um clima organizacional propício ao aprendizado e ao acolhimento de novas ideias (Fiuza, 2010); investem em ações ligadas ao aprendizado, compartilhamento de ideias e experiências entre os gestores da organização ou externos a ela (Rumke et al., 2016); permitem ao gestor visualizar gargalos (técnicos e comportamentais) no seu desempenho, podendo transformar a sua gestão em inovadora (Kachanakova, 2013); investem, principalmente, em reconhecimentos simbólicos como gratificação às inovações dos gestores (Bratton \& Gold, 2012); e promovem a incorporação e o acesso de novas tecnologias na organização, auxiliando na agilidade dos processos gerenciais inovadores (Horta \& Barbosa, 2017). A síntese da análise das cognições dos gestores confrontados com a literatura pode ser vista na Figura 1. 
Figura 1 - Síntese dos resultados

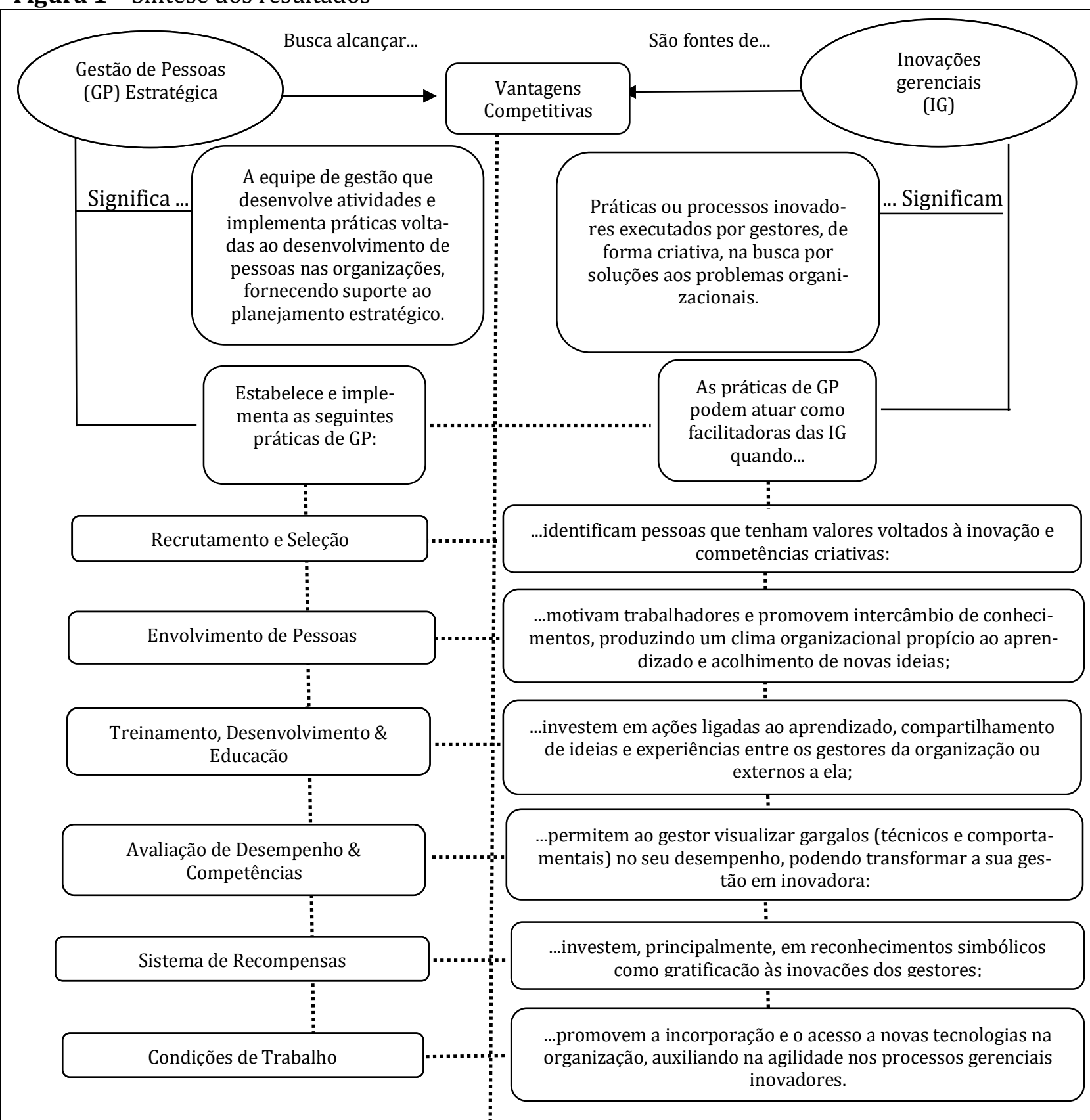

Principais IG implementadas por gestores como resultantes das práticas de GP:

- Administração do tempo, com vistas à otimização do trabalho das equipes, especialmente por meio da automatização de instrumentos de planejamento e controle de metas e resultados;

- Organização e aproximação das redes internas de relacionamentos (atividades lúdicas e reconhecimento simbólico) e externas à organização (benchmarking, busca de novas parcerias);

- Aperfeiçoamento da gestão participativa, priorizando a gestão da diversidade e composição de equipes multifuncionais, além da redistribuição e divisão de tarefas.

Fonte: Elaborado pelas autoras (2020).

A seção a seguir aborda o alcance do objetivo e as principais contribuições da pesquisa, as limitações do estudo e as sugestões para pesquisas futuras.

\section{Conclusão}

Esta pesquisa teve como objetivo analisar a relação entre práticas de gestão de pessoas e a implementação de inovações gerenciais a partir da cognição de gestores. Assim, ao acessar as cognições dos gestores, conhecendo a construção do pensamento que orienta as suas ações e decisões, foi possí- 
vel identificar que as práticas de GP atuam como facilitadoras das inovações gerenciais implementadas pelos gestores.

Este artigo contribui teoricamente com o campo da GP estratégica. Ficou evidente que quando a GP estabelece um planejamento de longo prazo alinhado aos objetivos organizacionais e implementa práticas específicas de gestão, colabora com a geração e implementação de inovações gerenciais. A pesquisa revelou a forma como cada uma das seis práticas de GP investigadas catalisam as inovações dos gestores. Ademais, o estudo evidenciou as inovações gerenciais, buscando esclarecer a concepção dos gestores enquanto agentes dessas inovações, bem como obtendo os principais exemplos de práticas e processos inovadores por eles executados. Identificou, também, que a relação entre práticas de GP e a implementação de inovações gerenciais perpassa três níveis de análise na dinâmica organizacional: macro (organizacional), meso (entre as lideranças e suas equipes) e micro (individual).

Destaca-se, ainda, o papel da técnica de coleta de dados adotada para a compreensão do fenômeno investigado, pois o uso de diferentes fases na coleta de informações representa grande contribuição para o campo dos estudos organizacionais, uma vez que a utilização de procedimentos que vão além de entrevistas semiestruturadas ainda é escassa nas pesquisas empíricas em Administração. Além disso, por se tratar de uma pesquisa que buscou acessar as cognições dos gestores, ou seja, a forma como esses percebem e constroem a realidade que experimentam nas organizações, a "técnica das fichas" foi essencial para que as pesquisadoras pudessem acessar as cognições dos entrevistados e alcançar os objetivos da pesquisa, uma vez os gestores entrevistados foram levados a refletir e organizar o pensamento a respeito do conteúdo que estava escrito em cada ficha. Ademais, o roteiro de entrevistas adicionado ao uso de fichas colaborou com a maior dinamicidade na coleta de informações.

Como contribuição para a prática gerencial, este estudo oferece à GP estratégica informações para que os gestores invistam em um planejamento estratégico com vistas a priorizar as práticas percebidas como mais relevantes à implementação de inovações gerenciais, que são: "envolvimento de pessoas" e "TD\&E", além de incrementar e remodelar aquelas percebidas como menos relevantes à implementação de inovações gerenciais, ou seja, "recrutamento e seleção", "AD\&C", "condições de trabalho" e, especialmente, "sistemas de recompensas".

Apesar das contribuições proporcionadas, destacam-se, a seguir, alguns questionamentos remanescentes após a conclusão desta pesquisa: que outros elementos da GP podem estar relacionados à implementação de inovações gerenciais? Como se dá a relação entre práticas de GP e inovações gerenciais em organizações pouco inovadoras? E em organizações públicas brasileiras? É possível mensurar a influência das inovações gerenciais nas práticas de GP? E das práticas de GP nas inovações gerenciais? Essas perguntas podem, certamente, orientar futuras pesquisas no campo da GP estratégica. Sugere-se, por fim, a realização de estudos com diferentes estratégias de coleta e análise de dados, como etnografia, estudo de caso e análise narrativa.

\section{Referências}

Armbruster, H., Bikfalvi, A., Kinkel, S., \& Lay, G. (2008). Organizational innovation: The challenge of measuring non-technical innovation in large-scale surveys. Technovation, 28(10), 644-657.

Bardin, L. (2011). Análise de conteúdo. São Paulo: Edições 70.

Barney, J. (1991). Firm resources and sustained competitive advantage. Journal of Management, 17(1), 99-120.

Bastos, A. V. B., Souza, J. J., Costa, V. M. F., \& Peixoto, A. L. A. (2011). A adoção de novas práticas de gestão: explorando o esquema cognitivo dos atores em empresas com diferentes padrões de inovação. Revista de Ciências da Administração, 13(31), 243-268.

Bianchi, E. M. P. G., Quishida, A., \& Foroni, P. G. (2017). Atuação do líder na gestão estratégica de pessoas: Reflexões, lacunas e oportunidades. Revista de Administração Contemporânea, 21(1), 41-61.

Birkinshaw, J., Hamel, G., \& Mol, M. J. (2008). Management Innovation. Academy of Management Review, 33(4), 825-845.

Boxall, P., \& Purcell, J. (2008). Strategy and Human Resource Management. (2. ed.). Nova York: Palgrave Macmillan.

Boxall, P. (2003). HR strategy and competitive advantage in the service sector. Human Resource Management Journal, 13(3), 5-20.

Bratton, J., \& Gold, J. (2012). Human resource management: teory and practice. Basingstoke: Palgrave Macmillan. 
Coradini, J. R., \& Murini, L. T. (2009). Recrutamento e seleção de pessoal: com agregar talentos à empresa. Disciplinarum Scientia/ Sociais Aplicadas, 5(1), 55-78.

Carvalho, L. F. N. (2004). Cognição em organizações: complexidade cognitiva e seus impactos no desempenho organizacional. Tese de doutorado, Fundação Getúlio Vargas, São Paulo, SP, Brasil.

Cruz, A. P. C. D., Frezatti, F., \& Bido, D. D. S. (2015). Estilo de liderança, controle gerencial e inovação: Papel das alavancas de controle. Revista de Administração Contemporânea, 19(6), 772-794.

Cunha, N. C., Palma, M., \& Santos, S. (2008). As práticas gerenciais promovendo a capacidade de inovação: estudo de múltiplos casos. Revista ADM. MADE, 12(3), 107-132.

Damanpour, F., \& Aravind, D. (2011). Managerial innovation: conceptions, processes, and antecedents. Management and Organization Review, 8, 423-454.

Damanpour, F. (2014). Footnotes to research on management innovation. Organization Studies, 35(9), 1265-1285.

Darwish, T. K., Wood, G., Singh, S., \& Singh, R. (2019). Human Resource Management in India: Performance and Complementarity. European Management Review.

Davenport, T. H., Prusak, L., \& Wilson, H. J. (2003). What's the big idea?: Creating and capitalizing on the best management thinking. Harvard Business Press.

Demo, G., Fogaça, N., Nunes, I., Edrei, L., \& Francischeto, L. (2011). Políticas de Gestão de Pessoas no Novo Milênio: Cenário dos Estudos Publicados nos Periódicos da Área de Administração entre 2000 e 2010. Revista de Administração Mackenzie, 12(5), 15-42.

Denzin, N., \& Lincoln, Y. (2006). O planejamento da pesquisa qualitativa: teorias e abordagens. Porto Alegre: Artmed.

Deus, L. F., \& Farias, J. S. (2015). A adoção do processo eletrônico de controle externo (e-TCU) no Tribunal de Contas da União: a experiência dos gestores envolvidos. RAI Revista de Administração e Inovação, 12(2), 270-292.

Dutra, J. D., \& Hipólito, J. A. M. (2012). Remuneração e recompensas. Rio de Janeiro: Elsevier.

Ekwoaba, J., Ikeije, U., \& Ufoma, N. (2015). The impact of recruitment and selection criteria on organizational performance. Global Journal of Human Resource Management, 3(2), 22-33.

Festa, M. P. (2015). Gestão e cultura intraempreendedora: um estudo sobre práticas gerenciais que promovem a inovação. Caderno Profissional de Administração da UNIMEP, 5(1), 41-58.

Fiuza, G. D. (2010). Políticas de gestão de pessoas, valores pessoais e justiça organizacional. Revista de Administração Mackenzie, 11(5), 56-81.

Fiske, S. T. \& Taylor, S. E. (1991). Social cognition 2nd ed. New York: McGraw-Hill.

Gomes, N. L., \& Farias, J. S. (2016). descentralização do processo de abastecimento em um hospital: Um estudo sob a ótica da Inovação. Amazônia, Organizações e Sustentabilidade, 4(2), 29-46.

Hamel, G. (2007). The Future of Management. Boston: Harvard Business School Publishing.

Horta, V., \& Barbosa, R. (2017). Desafios e barreiras à implantação da gestão do conhecimento em organizações. Biblionline, 13(2), 3-17.

Janissek, J., Aguiar, C. V. N., Mello, T. A. B., Ferreira, R. S., \& Campos, M. S. (2017). Práticas inovadoras de gestão no contexto das universidades públicas brasileiras: validação da escala para medir seu grau de importância e adoção. Revista do Serviço Público, 68(2), 259-284.

Kachanakova, A. (2013). Present state of performance evaluation in public administration organizations operating in Slovakia. Economic Annals-XXI, Slovakia.

Khosravi, P., Newton, C., \& Rezvani, A. (2019). Management innovation: A systematic review and metaanalysis of past decades of research. European Management Journal, 37(6), 694-707.

Lam, A. (2006). Organizational innovation. In Fagerberg, J.; Mowery, D.; Nelson, R (Eds.), The Oxford Handbook of Innovations. Oxford: Oxford University Press. p. 115-147.

Legge, K. (2005). Human resource management: rethorics and realities. Nova York: Palgrave Macmillan.

Lin, H., \& Su, J. (2014). A case study on adoptive management innovation in China, Journal of Organizational Change Management, 27(1), 83-114.

Lopes, D. P. T. (2017). Inovação gerencial na perspectiva da gestão de recursos humanos. (Tese de doutorado). Universidade Federal De Minas Gerais, Belo Horizonte, MG, Brasil.

Lu, K., Zhu, J., \& Bao, H. (2015). High-performance human resource management and firm performance: the mediating role of innovation in China. Industrial Management \& Data Systems, 115(2), 353-381.

Marx, R., Soares, J., \& Barros, L. (2016). Variáveis de contexto organizacional a serem consideradas no 
projeto de sistemas de recompensas orientados à inovação de produtos. Revista Brasileira de Gestão de Negócios, 18(60), 267-289.

Mol, M. J., \& Birkinshaw, J. (2009). The sources of management innovation: When firms introduce new management practices. Journal of Business Research, 62(12), 1269-1280.

Ofori, D., \& Aryeetey, M. (2011). Recruitment and selection practices in small and medium enterprises: Perspectives from Ghana. International Journal of Business Administration, 2(3), 45.

Penrose, E. T. (1959). The Theory of the Growth of the Firm. New York: Wiley.

Pinho, A. (2009). Comprometimento, entrincheiramento e consentimento organizacionais: uma análise destes vínculos, entre gestores e trabalhadores, de diferentes organizações. Tese de doutorado, Universidade Federal da Bahia, Salvador, Bahia, Brasil.

Pinho, A. P. M., \& Souza, J. (2019). Cognição e ação: Explicando as Bases Cognitivas do Comportamento Gerencial. In Tupinambá, A. C. R. \& Rodríguez, F. G. (Eds.). Liderança e Empreendedorismo em Perspectiva Intercultural (pp. 219-256). Fortaleza: Edições UFC.

Pinho, A. P. M., Silva, M. R. R., \& Evangelista, N. (2020). Cognições gerenciais sobre práticas gerenciais inovadoras e gestão de pessoas. Revista de Psicologia da UFC, 11(1), p. 49-58.

Pradana, M., Pérez-Luño, A., \& Fuentes-Blasco, M. (2020). Innovation as the key to gain performance from absorptive capacity and human capital. Technology Analysis \& Strategic Management, 1-13.

Prato Neto, C. R., dos Santos, B. M., Wegner, R. D. S., Marzall, L. F., Martinelli, M., \& Godoy, L. P. (2016). A importância do processo de recrutamento e seleção de pessoas na retenção do capital intelectual. Revista Espacios, 37 (32).

Ramos, É. N. P., \& Helal, D. H. (2010). A prática da gestão do conhecimento em uma empresa familiar do ramo varejista em Minas Gerais (MG): um estudo de caso. JISTEM: Journal of Information Systems and Technology Management, 7(2), 433-452.

Rogers, E. M. (1995). Diffusion of innovations. New York: The Free Press.

Rumke, B. D. P., Ramos, D., Fabricio, A., Obregon, S. L., \& Lopes, L. F. D. (2016). Levantamento das necessidades e elaboração de um plano de treinamento e desenvolvimento para uma empresa de TI. Revista de Carreiras e Pessoas, 6(2), 188-205.

Schreiber, D., \& Pinheiro, I. A. (2016). Analysis of the Innovative Practices at Construction Industry. Contextus - Revista Contemporânea de Economia e Gestão, 14(2), 6-35.

Schneider, S. C., \& Angelmar, R. (1993). Cognition in organizational analysis: Who's minding the store? Organization Studies, 14(3), 347-374.

SEBRAE - Serviço Brasileiro de Apoio às Micro e Pequenas Empresas (2013). Anuário do Trabalho na

Micro e Pequena Empresa. DIEESE - Departamento Intersindical de Estatística e Estudos Socioeconômicos, Brasília, DF. Disponível em: https://www.sebrae.com.br/Sebrae/Portal\%20Sebrae/Anexos /Anuario\%20do\%20Trabalho\%20Na\%20Micro\%20e\%20Pequena\%20Empresa_2013.pdf. Acesso em: 5 out. 2020.

Tan, C. L., \& Nasurdin, A. M. (2011). Human resource management practices and organizational innovation: assessing the mediating role of knowledge management effectiveness. Electronic journal of knowledge management, 9(2), 155.

Teece, D. J., Pisano, G., \& Shuen, A. (1997). Dynamic capabilities and strategic management. Strategic Management Journal, 18(7), 509-533.

Ulrich, D. (1998). Campeões de Recursos Humanos. São Paulo: Futura.

Ulrich, D., \& Dulebohn, J. H. (2015). Are we there yet? What's next for HR?. Human Resource Management Review, 25(2), 188-204.

Vaccaro, I. Jansen, J., Bosch, F., \& Volberda, H. (2012). Management innovation and leadership: the moderating role of organizational size. Journal of Management Studies, 49(1), 28-51.

Volberda, H. W., Van Den Bosch, F. A., \& Heij, C. V. (2013). Management innovation: Management as fertile ground for innovation. European Management Review, 10(1), 1-15.

Wright, P. M., \& McMahan, G. C. (1992). Theoretical perspectives for strategic human resource management. Journal of Management, 18(2), 295-320.

Zanelli, J. C. (2010). Estresse nas organizações de trabalho. São Paulo: Artmed. 